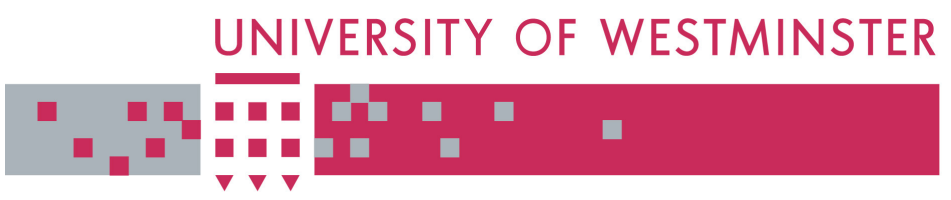

\title{
WestminsterResearch
}

http://www.wmin.ac.uk/westminsterresearch

\section{On the structure, convergence and performance of an adaptive I/Q mismatch corrector.}

\author{
Ediz Cetin \\ Izzet Kale \\ Richard Morling
}

Cavendish School of Computer Science

Copyright (C [2002] IEEE. Reprinted from 2002 IEEE 56th Vehicular Technology Conference proceedings :24-28 September 2002, Vancouver, Canada : VTC2002Fall Vancouver :pathway to ubiquitous wireless communications, pp. 2288-2292.

This material is posted here with permission of the IEEE. Such permission of the IEEE does not in any way imply IEEE endorsement of any of the University of Westminster's products or services. Internal or personal use of this material is permitted. However, permission to reprint/republish this material for advertising or promotional purposes or for creating new collective works for resale or redistribution must be obtained from the IEEE by writing to pubs-permissions@ieee.org. By choosing to view this document, you agree to all provisions of the copyright laws protecting it.

The WestminsterResearch online digital archive at the University of Westminster aims to make the research output of the University available to a wider audience. Copyright and Moral Rights remain with the authors and/or copyright owners. Users are permitted to download and/or print one copy for non-commercial private study or research. Further distribution and any use of material from within this archive for profit-making enterprises or for commercial gain is strictly forbidden.

Whilst further distribution of specific materials from within this archive is forbidden, you may freely distribute the URL of WestminsterResearch.

(http://www.wmin.ac.uk/westminsterresearch).

In case of abuse or copyright appearing without permission e-mail wattsn@wmin.ac.uk. 


\title{
ON THE STRUCTURE, CONVERGENCE AND PERFORMANCE OF AN ADAPTIVE I/Q MISMATCH CORRECTOR
}

\author{
Ediz Çetin, Izzet Kale and Richard C. S. Morling \\ University of Westminster, Department of Electronic Systems, \\ Applied DSP and VLSI Research Group, \\ London W1W 6UW, United Kingdom
}

\begin{abstract}
The $\mathrm{I} / \mathrm{Q}$ mismatches in quadrature radio receivers results in finite and usually insufficient image rejection, degrading the performance greatly. In this paper we present a detailed analysis of the Blind-Source Separation (BSS) based mismatch corrector in terms of its structure, convergence and performance. The results indicate that the mismatch can be effectively compensated during the normal operation as well as in the rapidly changing environments. Since the compensation is carried out before any modulation specific processing, the proposed method works with all standard modulation formats and is amenable to low-power implementations.
\end{abstract}

Keywords—phase and gain errors, $I / Q$ mismatch, blind source separation, adaptive signal processing, digital receivers.

\section{INTRODUCTION}

The I/Q mismatches in quadrature radio transceivers limit the image rejection (IR) that can be achieved resulting in a finite IR, degrading the bit-error-rate (BER) [1]-[7]. In general, it is difficult to eliminate circuit mismatches and obtain a high image-rejection ratio. For optimal system operation, wideband IR is required for a broad range of operating temperatures and signal power levels. This is not achievable using a one-time optimisation technique, such as trimming. Several adaptive techniques have been proposed to estimate and compensate the quadrature receiver errors [1][7]. In [4] and [5] the Gram-Schmidt orthogonalisation procedure is proposed for correcting the I/Q errors by using test/pilot signals. In [1] and [2] an interference cancellation based adaptive I/Q corrector is proposed. In this paper we carry out a detailed analysis of the non-data/pilot aided BSS based imbalance compensator developed for the quadrature receivers in [7].

The paper is organized as follows: Section II defines the model of the gain and phase imbalance compensator. Section III describes the performance analysis and the simulation results, while concluding remarks are given in Section IV.

\section{BSS-BASED I/Q CORRECTOR}

\section{A. Effects of $I / Q$ Errors}

In this section we will briefly describe the effects of phase and gain mismatches of the analog front-end. PSK modulated signal can be represented as:

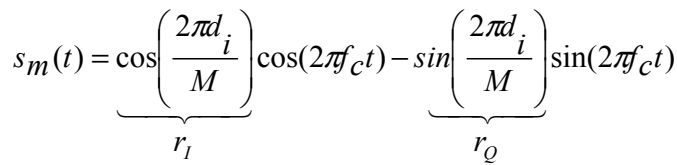

where, $d_{i}$ is the $M$-ary data, $d_{i} \in\{0,1, \ldots, M-1\}$. However, in the presence of analog front-end impairments the erroneous received baseband I and Q signals can be expressed as:

$$
\begin{aligned}
& r_{I}=g_{1} \cos \left(\frac{\varphi_{\varepsilon}}{2}\right) \cos \left(2 \pi f_{c} t\right)+g_{1} \sin \left(\frac{\varphi_{\varepsilon}}{2}\right) \sin \left(2 \pi f_{c} t\right) \\
& r_{Q}=g_{2} \sin \left(\frac{\varphi_{\varepsilon}}{2}\right) \cos \left(2 \pi f_{c} t\right)+g_{2} \cos \left(\frac{\varphi_{\varepsilon}}{2}\right) \sin \left(2 \pi f_{c} t\right)
\end{aligned}
$$

where $g_{1}=\left(1+0.5 \alpha_{\varepsilon}\right), g_{2}=\left(1-0.5 \alpha_{\varepsilon}\right)$ and $\varphi_{\varepsilon}$ and $\alpha_{\varepsilon}$ represent the phase and gain mismatches respectively. In the ideal case the I and Q channels are not correlated however in the presence of phase and gain errors there is a correlation between them. The cross-correlation, in the presence of phase and gain errors, at zero-lag is given in (3) and shown in Fig. 1.

$C_{r_{I} r_{Q}}(0)=\frac{1}{2} g_{1} g_{2} \sin \varphi_{\mathcal{E}}$

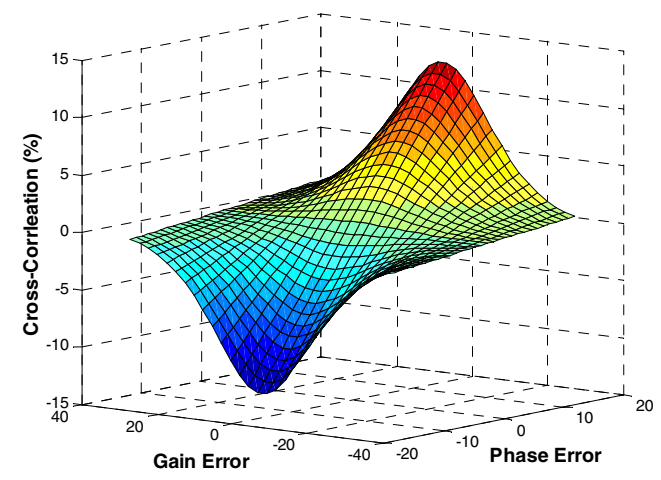

Figure 1 Cross-correlation Function

As it can be seen from Fig. 1, amount of phase and gain errors determine the cross-correlation between the I and Q channels.

\section{B. Application of BSS to $I / Q$ Correction}

In the theoretical derivation of the algorithm the following notations will be used:

$$
\begin{aligned}
& \text { Transmitted I/Q Signals: } \quad \mathbf{s}(z)=\left[s_{I}(z) s_{Q}(z)\right]^{T} \\
& \text { Received I/Q Signals: } \quad \mathbf{r}(z)=\left[\begin{array}{ll}
r_{I}(z) & r_{Q}(z)
\end{array}\right]^{T} \\
& \text { Corrected I/Q Signals: } \quad \mathbf{c}(z)=\left[\begin{array}{ll}
c_{I}(z) & c_{Q}(z)
\end{array}\right]^{T} \\
& \text { Mixing Vector: } \quad \mathbf{H}_{i}^{[k]}(z)=\left[h_{i}^{[k]}(0) \ldots h_{i}^{[k]}\left(L_{i}\right)\right]^{T} \\
& \text { Coefficient Vector: } \quad \mathbf{W}_{i}^{[k]}(z)=\left[w_{i}^{[k]}(0) \ldots w_{i}^{[k]}\left(L_{i}\right)\right]^{T}
\end{aligned}
$$

where $L_{i}$ is the filter order and $i=1,2$. In the presence of I and $\mathrm{Q}$ phase and gain mismatches the received signal $\mathbf{r}[\mathrm{k}]$ can be expressed as [7]: 


\section{$\mathbf{r}[k]=\mathbf{H s}[k]$}

where $\mathbf{H}$ is the unknown non-singular mixing matrix which is determined by the phase and gain errors [7] and $\mathbf{s}[k]$ is the transmitted signal. Given the received vector $\mathbf{r}[\mathrm{k}]$, the source separation problem comprises the recovery of the original signals in an unsupervised way by finding a de-mixing matrix $\mathbf{W}$ hence recovering the sources:

$$
\begin{aligned}
\mathbf{c}[k] & =\mathbf{W r}[k] \\
& =\mathbf{W H s}[k]
\end{aligned}
$$

Application of the BSS to the I/Q problem is depicted in Fig 2.

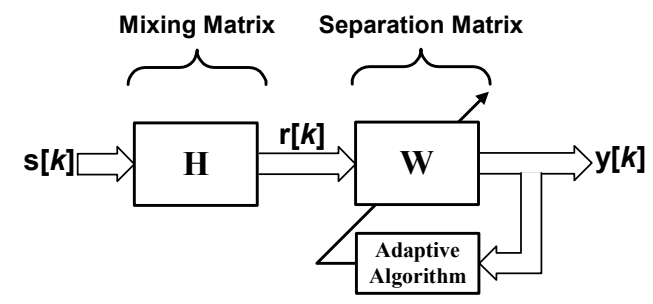

Figure 2 Application of the BSS to I/Q correction

In the next section the structures for the I/Q channel phase and gain error compensation will be looked at

\section{Structures for the Solution}

In this section we will first derive the general properties of the solutions to the $\mathrm{I} / \mathrm{Q}$ phase and gain correction before discussing any specific criterion or adaptive method. The only assumption we make is that the transmitted signals, $s_{\mathrm{I}}[k]$ and $s_{\mathrm{Q}}[k]$ are orthogonal and not correlated witch each other. Hence, this assumption implies that:

$E\left[s_{I}[k] s_{Q}[k-n]\right]=0 \quad \forall n$

Possible feed-forward solution to the source separation problem is depicted in Fig 3.

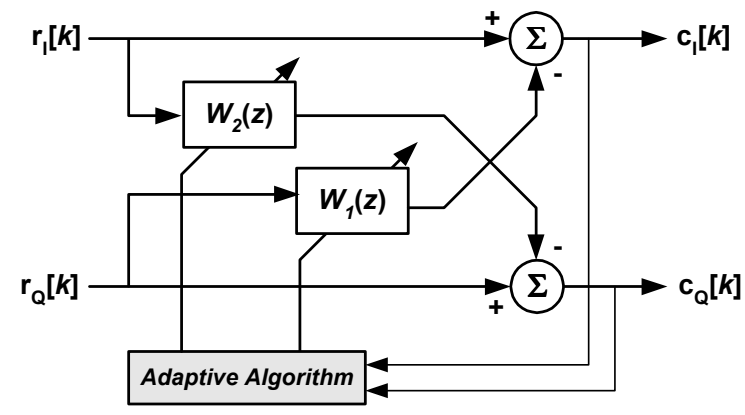

Figure 3 BSS-based I/Q corrector [7]

For the feed-forward case the source estimates, $\mathrm{c}_{\mathrm{I}}(z)$ and $\mathrm{c}_{\mathrm{Q}}(z)$, become:

$$
\begin{aligned}
& c_{I}(z)=\left(1-W_{1}(z) H_{2}(z)\right) s_{I}(z)+\left(H_{1}(z)-W_{1}(z)\right) s_{Q}(z) \\
& c_{Q}(z)=\left(H_{2}(z)-W_{2}(z)\right) s_{I}(z)+\left(1-W_{2}(z) H_{1}(z)\right) s_{Q}(z)
\end{aligned}
$$

When the filters converge, i.e. $W_{1}(z)=H_{1}(z)$ and $W_{2}(z)=H_{2}(z)$ then the source estimates become:

$$
c_{I}(z)=\left(1-H_{1}(z) H_{2}(z)\right) s_{I}(z)
$$$$
c_{Q}(z)=\left(1-H_{1}(z) H_{2}(z)\right) s_{Q}(z)
$$

As it can be seen from (8) the I and Q channels have the same gain and are orthogonal again. Also, $\left(1-H_{1}(z) H_{2}(z)\right) \approx 1$ and can be safely ignored. An alternative implementation for the separation structure is found by placing the filters in the feedback loop. The structure is shown in Fig 4.

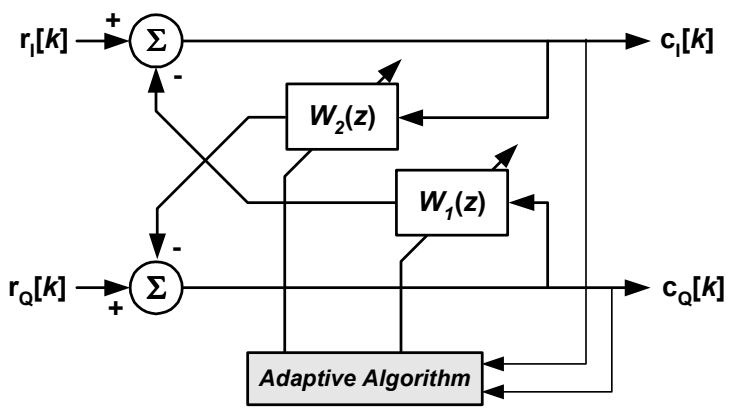

Figure 4 BSS-based I/Q corrector

For the feed-back case the source estimates, $\mathrm{c}_{\mathrm{I}}(z)$ and $\mathrm{c}_{\mathrm{Q}}(z)$, become:

$$
\begin{aligned}
& c_{I}(z)=\frac{1}{1-W_{1}(z) W_{2}(z)}\left[\left(1-W_{1}(z) H_{2}(z)\right) s_{I}(z)+\left(H_{1}(z)-W_{1}(z)\right) s_{Q}(z)\right] \\
& c_{Q}(z)=\frac{1}{1-W_{1}(z) W_{2}(z)}\left[\left(H_{2}(z)-W_{2}(z)\right) s_{I}(z)+\left(1-W_{2}(z) H_{1}(z)\right) s_{Q}(z)\right]
\end{aligned}
$$

When the filters converge, i.e. $W_{1}(z)=H_{1}(z)$ and $W_{2}(z)=H_{2}(z)$ then the source estimates become:

$$
\begin{aligned}
& c_{I}(z)=s_{I}(z) \\
& c_{Q}(z)=s_{Q}(z)
\end{aligned}
$$

As it can be seen from (10) the I and Q channels have the same gain and are orthogonal again. The filter $\mathrm{W}_{1}$ identifies the crosstalk from $\mathrm{Q}$ to I channel and the filter $\mathrm{W}_{2}$ identifies the crosstalk from I to $\mathrm{Q}$. These estimates are then subtracted to yield estimates of sources $s_{\mathrm{I}}[k]$ and $s_{\mathrm{Q}}[k]$ that are separated/uncorrelated

\section{Adaptation of $W_{1}(z)$ and $W_{2}(z)$}

In the previous section, while deriving the general properties of the structures for the I/Q correction, no specific criteria or adaptive method has been proposed for the adaptation of filters $\mathrm{W}_{1}(z)$ and $\mathrm{W}_{2}(z)$. In this section we look into the use of simple Least-Mean-Squares (LMS) algorithm [8] to update filter coefficients. The proposed algorithm is derived as an intuitive extension of the classical LMS Adaptive-Noise Canceller (ANC).

The proposed structure is re-drawn in Fig. 5 with two cross-coupled ANC's highlighted. The Mean Square Errors $\left(\xi_{1}\right.$ and $\left.\xi_{2}\right)$ can be expressed as:

$$
\begin{aligned}
& \xi_{1}=E\left[r_{I}{ }^{2}[k]\right]-2 \mathbf{P}_{1}^{T} \mathbf{W}_{1}+\mathbf{W}_{1}{ }^{T} \mathbf{R}_{1} \mathbf{W} \\
& \xi_{2}=E\left[r_{Q}{ }^{2}[k]\right]-2 \mathbf{P}_{2}{ }^{T} \mathbf{W}_{2}+\mathbf{W}_{2}{ }^{T} \mathbf{R}_{2} \mathbf{W}_{2}
\end{aligned}
$$

where $\mathbf{R}_{1}=\mathrm{E}\left[\mathbf{R}_{Q}[\mathrm{k}] \mathbf{R}_{Q}[k]^{T}\right]$ and $\mathbf{R}_{2}=\mathrm{E}\left[\mathbf{R}_{I}[\mathrm{k}] \mathbf{R}_{I}[k]^{T}\right]$ are the input auto-correlation matrices and $\mathbf{P}_{1}=\mathrm{E}\left[r_{I}[\mathrm{k}] \mathbf{R}_{Q}[k]\right]$ and $\mathbf{P}_{2}=\mathrm{E}\left[r_{Q}[\mathrm{k}] \mathbf{R}_{I}[k]\right]$ are the cross-correlation matrices. The criterion of the LMS adaptive noise cancellers, ANC 1 and ANC 2, is to minimize the energies $\xi_{1}[k]$ and $\xi_{2}[k]$ in the signal estimates $c_{\mathrm{I}}[k]$ and $c_{\mathrm{Q}}[k]$ respectively. 


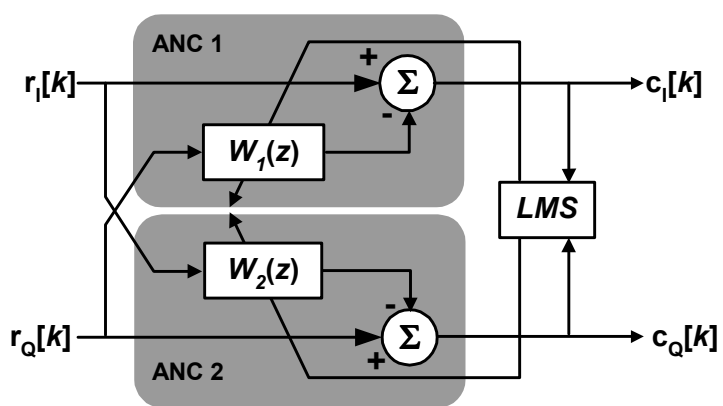

Figure 5 BSS-based I/Q corrector

An interesting property of the LMS algorithm is its decorrelation property [8]. When the algorithm has converged the signals $c_{\mathrm{I}}[k]$ and $r_{\mathrm{Q}}[k]$ as well as $c_{\mathrm{Q}}[k]$ and $r_{\mathrm{I}}[k]$ are decorrelated over the length of the adaptive filter [8]. This gives:

$$
\begin{aligned}
& E\left[c_{I}[k] r_{Q}[k-m]\right]=C_{c_{I} r_{Q}}[m]=0 \quad m=0, \ldots, L_{1} \\
& E\left[c_{Q}[k] r_{I}[k-n]\right]=C_{c_{Q} r_{I}}[n]=0 \quad n=0, \ldots, L_{2}
\end{aligned}
$$

Minimization of $\xi_{1}[k]$ and $\xi_{2}[k]$ thus equivalent to decorrelation of the signal estimates $c_{\mathrm{I}}[k]$ and $c_{\mathrm{Q}}[k]$ with the interference references $r_{Q}[k]$ and $r_{[}[k]$ respectively. To show this more rigorously, the mixing filter $\mathrm{H}_{2}(z)$ is first set to zero. The ANC 1 in Fig. 3 is then applied under the mild assumption that filters $\mathrm{H}_{1}(z)$ and $\mathrm{W}_{1}(z)$ are both of order $L_{1}$. First the expressions for the minimization are given as:

$$
\begin{aligned}
\xi_{1} & =E\left[s_{I}^{2}[k]\right]+\mathbf{W}_{1}^{T} \mathbf{R} \mathbf{W}_{1}-\mathbf{W}_{1}^{T} \mathbf{P} \\
\nabla \xi_{1} & =2 \mathbf{R} \mathbf{W}_{1}-2 \mathbf{P}=0
\end{aligned}
$$

Next expressions are derived for the decorrelation:

$$
\begin{aligned}
\mathbf{C}_{c_{I} r_{Q}} & =E\left[c_{I}[k] \mathbf{R}_{Q}[k]\right]=E\left[\left(r_{I}[k]-\mathbf{W}_{1}^{T} \mathbf{R}_{Q}[k]\right) \mathbf{R}_{Q}[k]\right] \\
& =\mathbf{P}-\mathbf{R} \mathbf{W}_{1}=0
\end{aligned}
$$

Comparing the above equations indeed shows the equivalence:

$$
\mathbf{C}_{c_{I} r_{Q}}=0 \equiv \nabla \xi=0
$$

As a conclusion we may say that the LMS algorithm is an adaptive decorrelation algorithm. Same analysis applies to the ANC 2 in the bottom branch as well. As illustrated in Section $\mathrm{C}$, it is indeed possible to obtain the desired source estimates when the structure of Fig. 3 is used. The update equations are given as:

$$
\begin{array}{ll}
w_{1}{ }^{[k+1]}[m]=w_{1}{ }^{[k]}[m]+2 \mu_{1} c_{I}[k] r_{Q}[k-m] & m=0 \ldots L_{1} \\
w_{2}{ }^{[k+1]}[n]=w_{2}{ }^{[k]}[n]+2 \mu_{2} c_{Q}[k] r_{I}[k-n] & n=0 \ldots L_{2}
\end{array}
$$

where $L_{1}$ and $L_{2}$ are the filter orders. If the set of update equations (16) converge, $c_{\mathrm{I}}[k]$ and $c_{\mathrm{Q}}[k]$ will be decorrelated over the length of both filters [8]:

$$
\begin{array}{cc}
C_{c_{I} c_{Q}}[m]=E\left[c_{I}[k] c_{Q}[k-m]\right]=0 & m=0, \ldots, L_{1} \\
C_{c_{I} c_{Q}}[n]=E\left[c_{Q}[k] c_{I}[k-n]\right]=0 & n=0, \ldots, L_{2}
\end{array}
$$

The decorrelation property of the proposed algorithm can be shown in the frequency domain by using the frequency domain equivalent of the cross-correlations, the cross- spectrum. The expression for zero cross-spectrum of the signal estimates yields:

$$
\begin{aligned}
\Phi_{c_{1} c_{Q}}(z) & =\left(1-W_{1}(z) H_{2}(z)\right)\left(H_{2}\left(z^{-1}\right)-W_{2}\left(z^{-1}\right)\right) \Phi_{s 1 s 1}(z) \\
& +\left(1-W_{2}\left(z^{-1}\right) H_{1}\left(z^{-1}\right)\right)\left(H_{1}(z)-W_{1}(z)\right) \Phi_{s 2 s 2}(z) \\
& =0 \quad \forall z
\end{aligned}
$$

Hence, decorrelation is indeed a necessary condition for signal separation and restoration of the orthogonality and equal amplitudes of the I and Q channels.

\section{PERFORMANCE EVALUATION}

To analyse the performance of the proposed structure, we consider linearly modulated communications signals, namely M-PSK and M-QAM with ideal symbol rate sampling. We assume an AWGN and Rayleigh Fading channels and phase and gain errors of $30^{\circ}$ and $6 \mathrm{dBs}$ respectively.

The performance of the adaptive algorithm is characterised by the Modelling-ERRor (MERR). This gives a global figure for the quality of the identification of the coupling filters $H_{1}(z)$ and $H_{2}(z)$ by $W_{1}(z)$ and $W_{2}(z)$. The curve of the MERR versus time (or frames) shows the modelling performance of the proposed algorithm. What is more, the MERR can be used to observe the convergence rate and/or the steady state performance of the proposed adaptive system. The MERR is defined as the squared norm of the difference of the transfer functions between the original filters used in the mixture and the estimated filters, relative to the squared norm of the mixture filter. It is given as:

$$
\operatorname{MERR}_{w_{i}}=\frac{\left|H_{i}(z)-W_{i}(z)\right|^{2}}{\left|H_{i}(z)\right|^{2}}
$$

In the time domain it is defined as the expected value of the sum of squares of the difference between the original and the estimated filters. It is expressed as follows:

$$
\varepsilon_{i}[k]=\frac{E\left[\sum_{l=0}^{L i}\left(h_{i}[l]-w_{i}^{k}[l]\right)^{2}\right]}{\sum_{l=0}^{L i} h_{i}^{2}[l]}
$$

where $L_{i}$ is the filter order $(i=1,2)$. First we will investigate the influence of the filter order $\left(L_{i}\right)$ on the performance of the proposed solution. The filter order cannot be chosen arbitrarily small. There must be sufficient degrees of freedom to model the unknown impulse responses $h_{1}[k]$ and $h_{2}[k]$. Fig. 6 depicts the modelling error for different filter tap lengths $\left(l_{i}=L_{i}+1\right)$ using 16-PSK modulated signals.
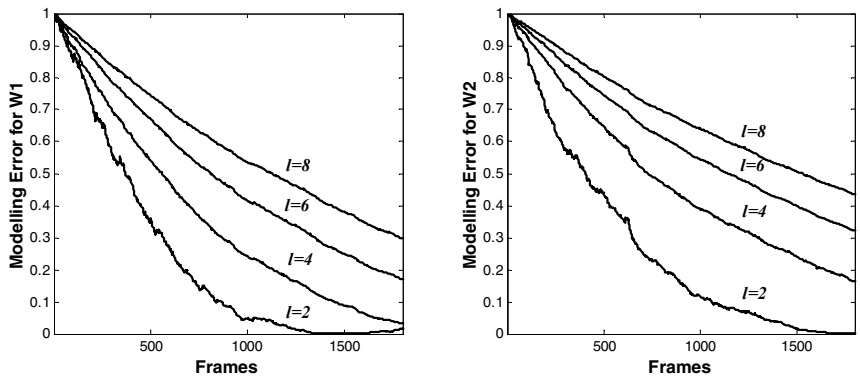

Figure 6 Modelling Error for different filter tap length $l_{i}$ 
From Fig. 6 we can see that, longer filters converge slower. What is more, increase of the filter tap lengths leads to larger misadjustment as expected [8]. Hence, filter order of 1 (i.e. $l_{i}$ $=2$-taps) is chosen for the proposed algorithm. Modelling error for different step-sizes $(\mu)$ using 16-PSK modulated signals is shown in Fig. 7.

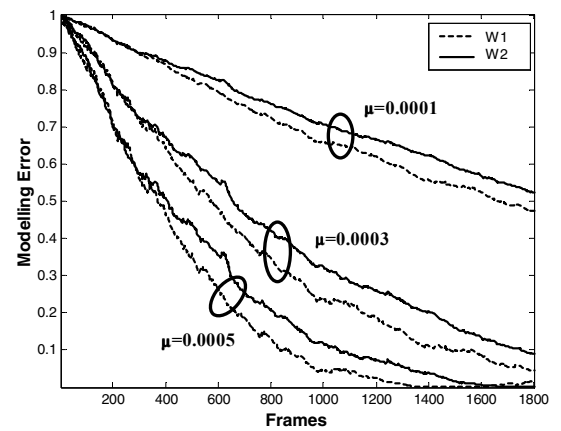

Figure 7 Modelling Error for different filter length $L_{i}$

Step-size values, smaller than 0.0005 and larger than 0.001 , that made the system unstable were discarded from the Fig. 7. As it can be seen from Fig. 7 step-size, $\mu=0.0005$, gives the best performance.

We now concentrate on the application of the proposed algorithm to different modulation formats. We consider two cases: (i) 32-PSK with an SNR of 32dB and (ii) 16-QAM with an SNR of 20dB. Figs 8 and 9 depict the constellation and eye diagrams for the application of the BSS-based corrector to 32$\mathrm{PSK}$ for an $\mathrm{SNR}=32 \mathrm{~dB}$ and 16-QAM for an $\mathrm{SNR}=20 \mathrm{~dB}$.

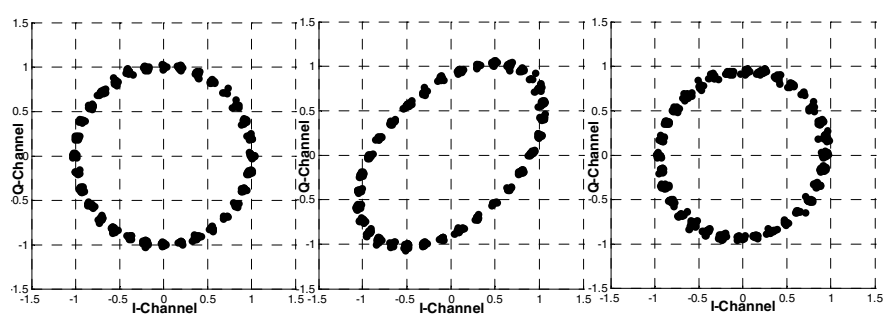

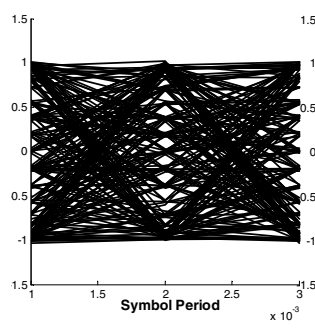

(a)

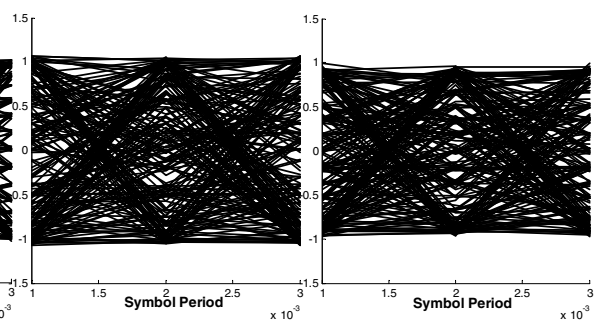

(b) (c)
Figure 8 Constellation and Eye Diagrams (a) Original, (b) With phase and gain error and (c) Corrected, for 32-PSK modulated signals

As it can be seen, the erroneous constellation and eye diagrams of Fig. 8 (b) are transformed (c) almost matching the ideal diagrams of $(\mathrm{a})$.
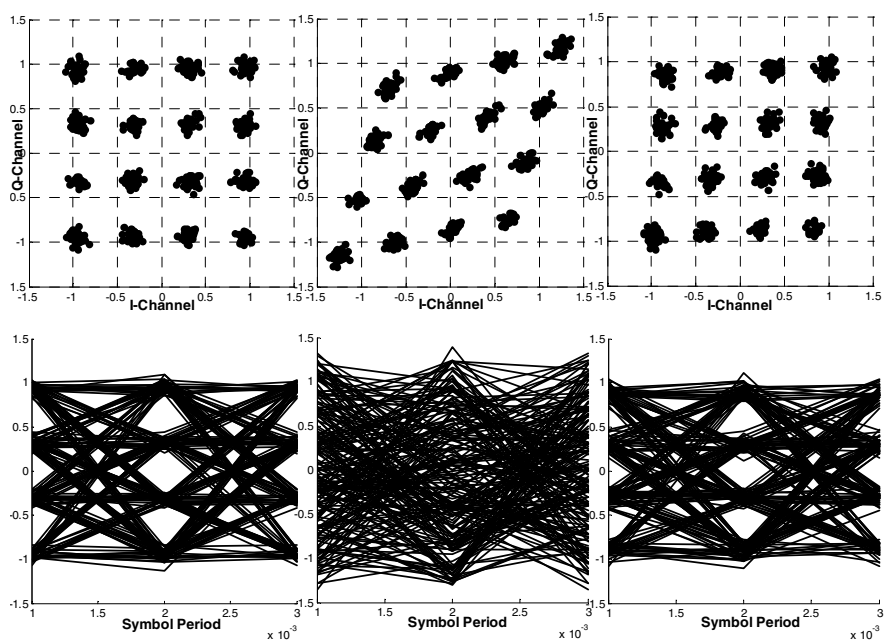

(a)

(b)

(c)

Figure 9 Constellation and Eye Diagrams (a) Original, (b) With phase and gain error and (c) Corrected, for 16-QAM modulated signals

Once again, the erroneous constellation and eye diagrams of (b) are transformed to those of (c) almost matching the ideal diagrams of (a). Fig. 10 depicts the modelling error for both of the above cases.

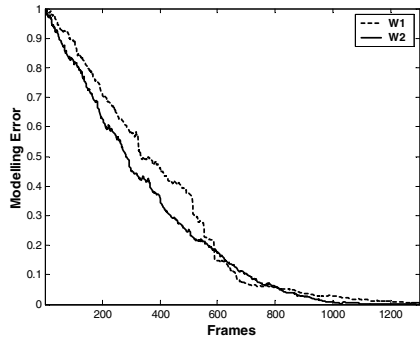

(a)

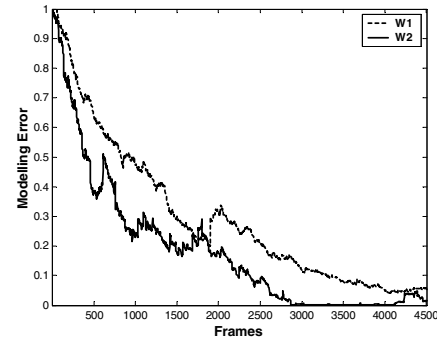

(b)
Figure 10 Modelling Error for (a) 32-PSK and (b) 16-QAM

As it can be seen, de-mixing filters $\mathrm{W}_{1}(z)$ and $\mathrm{W}_{2}(z)$ almost match the mixing filters $\mathrm{H}_{1}(z)$ and $\mathrm{H}_{2}(z)$; hence the modelling errors are almost zeroed starting from frames 1100 for (a) and 4000 for (b).

Another performance measure is the capability of the adaptive algorithm in tracking non-stationary environments i.e. time varying and frequency dependent phase and gain errors. In order to show the robustness of the proposed approach we start by adapting the filters to the $30^{\circ}$ phase and $6 \mathrm{~dB}$ of gain error. We then switch the phase error to $15^{\circ}$ and the gain error to $3 \mathrm{~dB}$. This enables us to examine the tracking behaviour of the structure to the reaction to phase and gain error changes. Fig. 11 depicts the tracking capability of the proposed algorithm. 


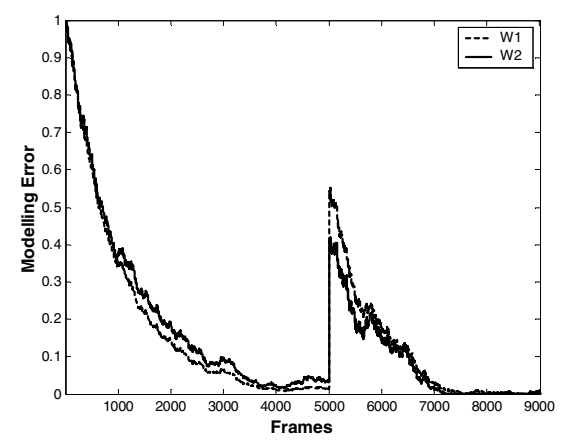

Figure 11 Tracking Capability

As it can be seen from the Fig. 11, the modelling error is almost zeroed from 4000 frames then we change the phase and gain errors to $15^{\circ}$ and $3 \mathrm{~dB}$ respectively. The filters track the changes rapidly and the modelling error is once again zeroed after 2000 frames. Hence the algorithm is capable of tracking the changes in the environment promptly.

Another performance measure is the capability of the adaptive algorithm in fading environments In order to show the robustness of the proposed approach we use Rayleigh Fading channel instead of the AWGN channel. Fig. 12 depicts the simulation results.

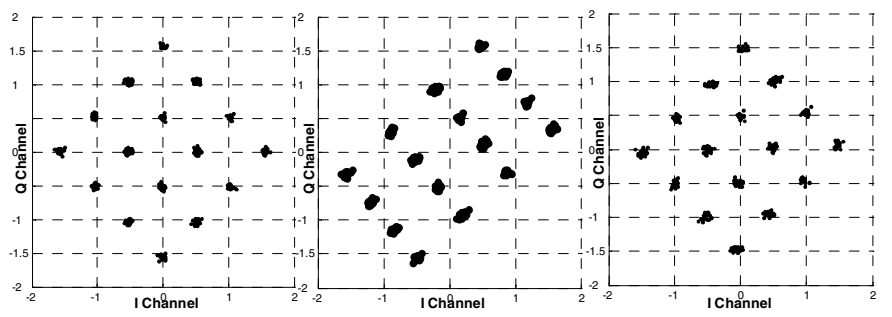

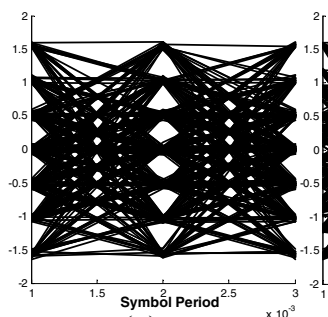

(a)

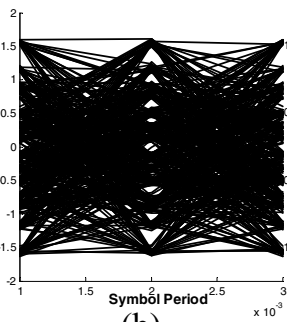

(b)

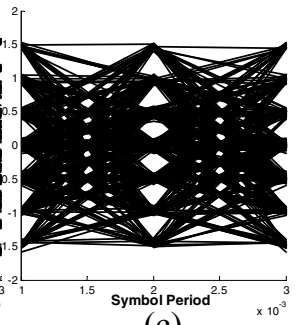

(c)
Figure 12 Constellation and Eye Diagrams (a) Original, (b) With phase and gain error and (c) Corrected, for 16-QAM modulated signals with Rayleigh Fading Channel.

As it can be seen from the constellation and eye diagrams of Fig. 12, the algorithm is capable of removing the phase and gain errors with fading channels without the need to carry out channel equalisation.

The modelling error for 16-QAM modulated signal with Rayleigh Fading Channel and phase and gain errors of $30^{\circ}$ and $6 \mathrm{~dB}$ respectively is depicted in Fig. 13. As it can be seen from Fig. 13 the proposed algorithm is able to identify and closely model the mixing filter impulse responses $h_{1}[k]$ and $h_{2}[k]$. The modelling error is almost zeroed after 950 frames.

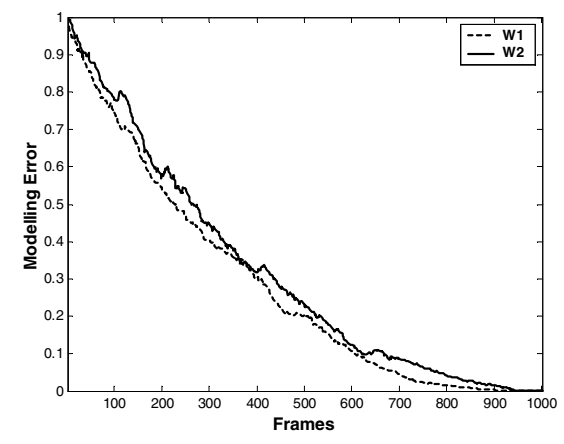

Figure 13 Modelling Error 16-QAM with fading channel

\section{CONCLUSIONS}

In this paper we have presented the simulation study of results derived analytically for a BSS centred I/Q mismatch compensator employing an LMS driven FIR filter set-up. The study examined our algorithm's performance for a variety of modulation formats, and reported on M-QAM and M-PSK in this paper. As the algorithm is independent of modulation scheme and format its applicability in different communications environments has been demonstrated, through numerous simulation experiments and only a few representative examples reported here. The algorithm should work with all standard modulation formats such as PAM, QAM, PSK, GMSK and OFDM. The algorithms ability to compensate and minimize the analog front-end non-linearities in rapidly changing environments has also been demonstrated. Furthermore, the algorithm's performance has been scrutinized under a noisy Rayleigh Fading channel environment and has been demonstrated to work satisfactorily. As the adaptive scheme reported in this paper has a very low computational complexity, it is amenable to low-complexity, low-power software/custom hardware implementations.

\section{REFERENCES}

[1] Li Yu; Snelgrove W.M., "A novel adaptive mismatch cancellation system for quadrature IF radio receivers" IEEE Transactions on Circuits and Systems II: Analog and Digital Signal Processing, vol. 46 issue 6, pp. $789-801$, June 1999

[2] Valkama, M.; Renfors M., "Advanced DSP for I/Q imbalance compensation in a low-IF receiver", IEEE International Conference on Communications (ICC 2000), vol. 2, vol.2, pp. $768-772,2000$

[3] Lohtia, A., Goud, P., Englefield, C., "An adaptive digital technique for compensating for analog quadrature modulator/demodulator impairments", IEEE Pacific Rim Conference on Communications, Computers and Signal Processing, vol. 2, pp. 447-450, 1993

[4] Churchill F.E., G.W. Ogar and B.J. Thompson, "The Correction of I and Q Errors in a Coherent Processor", IEEE Trans. on Aerospace and Electronic Systems, vol. AES-17, no.1, pp. 131-137, January 1981.

[5] Huang, X.; Caron, M.; Hindson, D., "A recursive Gram-Schmidt orthonormalization procedure and its application to communications" IEEE Third Workshop on Signal Processing Advances in Wireless Communications (SPAWC '01), pp. $340-343,2001$

[6] McLeod, MD, "Fast calibration of IQ digitiser systems", IEE Colloquium on system aspects and applications of ADCs for radar, sonar and communications, pp. 1-4, November 1987

[7] Cetin, E.; Kale, I.; Morling, R.C.S., "Adaptive digital receivers for analog front-end mismatch correction", IEEE VTS $54^{\text {th }}$ Vehicular Technology Conference (VTC 2001 Fall), vol: 4, pp. 2519-2522, 2001

[8] Widrow B. and S.D. Stearns, "Adaptive Signal Processing", Prentice Hall, 1985 ISBN: 0-13-004029-0 